\title{
IMPLEMENTATION OF THE GLOBAL AERONAUTICAL DISTRESS AND SAFETY SYSTEM (GADSS)
}

\author{
Dimov Stojce ILCEV \\ Durban University of Technology, Durban, South Africa \\ Received 08 May 2018; accepted 24 June 2018
}

\begin{abstract}
In this paper is introduced the first proposal for development of Global Aeronautical Distress and Safety System (GADSS) in 1999 by the author of this article. The GADSS is de facto the integration of space (radio and satellite) Communication, Navigation and Surveillance (CNS) with Tracking, Detecting and Search and Rescue (SAR) systems, which have to provide airmen with global communications and locating networks. The GPS, GLONASS and other Global Navigation Satellite Systems (GNSS) provide precise positioning data for vessels, land vehicles and aircraft, but modern CNS demands need for enhanced services and augmentation of GNSS networks. Both networks have to be integrated under an GADSS umbrella with elements capable of being operated by any individual onboard aircraft to ensure prompt distress alert for SAR procedure. The enhanced concept of GADSS is that SAR authorities ashore and ships in the immediate vicinity of the aircraft in distress have to be rapidly alerted via radio and satellite communication systems and to assist in a coordinated SAR operations with the minimum of delay. In 2016, 16 years in delay, the International Civil Aviation Organization (ICAO) has begun its process to amend international standards and recommended practices to align with GADSS concept. This paper will also introduce the necessary networks and equipment, which has to ensure harmonized and enhanced maritime and aeronautical global SAR systems.
\end{abstract}

Keywords: GADSS, GNSS, CNS, SAR, ICAO, VDL, RADS-B, SDL, SADS-B, ELT, EPIRB, PLB, GAT.

\section{Introduction}

The new Global Aeronautical Distress and Safety System (GADSS) is going to be an international network that uses improved terrestrial and satellite communication technology, including onboard airborne radio and satellite communication systems. It will ensure rapid distress alerting of shore-based rescue and safety communications authorities in the event of aircraft emergency at sea, on the ground and in the air. As stated above, the GADSS network was proposed by the author of this paper, which has to be developed providing airmen with global communications, positioning and locating networks, enhanced safety, security, distress alert, tracking and detecting facilities.

Both networks have to be integrated under an GADSS umbrella with elements capable of being operated by and an individual onboard aircraft with minimum communication knowledge, even they are crew staff, and yet enable alerting with Search and Rescue (SAR) services to be reliably coordinated. This system will be similar to the Global Maritime Distress and Safety System (GMDSS) developed by IMO and became effective on 1 February 1999. It has to be of prime importance to all aeronautical personnel for it is likely that elements within the GDASS will affect every individual in the future. This also has to be very important for aircraft crew, because in case that pilot is unable to provide distress alert, it can be conducted by any crewmember for saving of crew and passenger lives. The major importance of the GDASS will be to provide enhanced Safety of Lives in Distress (SOLID) of aircraft incidents at sea and on the ground.

The author of this paper wrote for the first time about his invention GDASS in 2000 and then explained about in his book "Global Mobile Satellite Communications" published by Springer in January 2005. He also wrote an entire chapter about GADSS in his two volume books "Global Aeronautical Communication, Navigation and Surveillance (CNS)" published by AIAA in 2013. Since 2000 the ICAO administration never reply on his letter regarding eventual collaboration in research and development of GADSS network. Problem is that ICAO proposed the GADSS in 2016, and still is not clear what technologies will use new GADSS network (Ilcev, 2013, 2017).

*Corresponding author. E-mail: ilcev@dut.ac.za 


\section{Termination of Future Air Navigation System (FANS)}

Traditionally, aircraft is subject to the current ICAO Convention and Regulation relating to the aeronautical communication and navigation. Thus, long time ago ICAO developed the Future Air Navigation System (FANS), which until recently was attribute as a total failure, so it has to be abandoned in the near future. As stated earlier, author of this paper many times since 2000 proposed GADSS project to all ICAO offices, but they ignored his letters. However, in 2016 ICAO proposed GADSS project as their own system not respecting, recognizing and admitting author's invention.

However, CNS is a well-established acronym for new Communication, Navigation and Surveillance, which together with proposed GADSS will be de facto the future real systems as successors to long term FANS scenario. Accordingly, in the future will be necessary to provide some more effective aeronautical system for air-toground, ground-to-air and air-to-air commercial, safety and distress radio and satellite voice or data communications, navigation data and surveillance information. The synonym surveillance as a part of CNS system means radio and satellite surveillance and not radar surveillance only.

At present aircraft can use two kinds of onboard communication systems: old Traditional Communications VHF and HF radio and new satellite communications. Integration of these two types of aircraft space communications has to provide basis for development GADSS network. The main tasks of ICAO will be to initiate GADSS project with establishment group of experts and to produce guidelines for identifying system requirements, technologies and effectiveness.

The basic concept of the GADSS has to be technology transfer and innovations of new radio and satellite CNS equipment for safety and security, reliable distress alert and effective procedure of the SAR local and regional authorities. The SAR forces have to come quickly in the immediate vicinity at sea or on the land, and in the same time have rapidly to alert all possible rescue resources about position of distress incident. In such a way, all together can assist in a coordinate SAR operation with the minimum delay (Ilcev, 2011, 2013; Global Aeronautical Distress and Safety System, 2002).

\section{Basic Concept for Development of GADSS Project}

The Space Science Centre (SSC) at DUT leaded by author of this paper completed the proposal regarding the basic project concept for the development of the future GADSS network. First of all, it will be necessary to establish a Task Force Group (TFG) for development of GADSS project, which in some way will be similar to the current Global Maritime Distress and Safety System (GMDSS) developed by IMO and which became effective on 1 February 1999.
Before that, author of this paper worked onboard ships as master mariner using future GMDSS network and in 2000 he proposed to ICAO that they have to abandon their long term FANS and start with development of GADSS network. They didn't hear his voice and just last year ICAO started to talk about GADSS.

Similar to GMDSS, the new GMDSS network has to be divided in 4 sea areas:

I - The Area A1 (20 to $50 \mathrm{NM}$ ) in the VHF, UHF and VHF Digital Selective Call (DSC) coverage, which have to depend on coverage of each VHF Ground Radio Stations (GRS) or Air Traffic Control (ATC) and Air Traffic Management (ATM) airport communication facilities;

II - The Area A2 (50 to $400 \mathrm{NM}$ ) in the VHF, HF and Inmarsat coverage;

III - The Area $\mathrm{A} 3\left(70^{\circ} \mathrm{N}\right.$ to $\left.70^{\circ} \mathrm{S}\right)$ VHF, HF and Inmarsat Geostationary Earth Orbit (GEO), Medium Earth Orbit (MEO) or other Non-GEO mobile satellite coverages; and

IV - The Area A4 (Polar Areas above $70^{\circ} \mathrm{N}$ or S) VHF, HF, Iridium satellite Low Earth Orbit (LEO) and Highly Elliptical Coverage (HEO) or Russian Molniya satellite coverages.

The TFG board for development and implementation of GADSS network has to prepare project in which have to be included the following Radio and Satellite subnetworks:

1. Radio Subnetworks - They have to include HF, VHF and perhaps UHF radio systems, such as VHF, VHF DSC, VHF Data Link (VDL), Broadband VHF (B-VHF), Radio Automatic Dependent Surveillance-Broadcast (RADS-B), Radio Automatic Identification System (R-AIS), HF Data Link (HDL) and others;

2. Satellite Subnetworks - They have to integrate Inmarsat, Iridium and upgraded networks, such as Inmarsat Aeronautical Mobile Satellite Communications (AMSC), Aircraft Satellite Data Links (SDL), Satellite Automatic Dependent Surveillance-Broadcast (SADS-B), Iridium Data Link and so on;

3. Satellite Search and Rescue (SAR) Subnetworks These SAR subsystems integrate LEOSAR, MEOSAR and GEOSAR satellite networks as a part of international Cospas-Sarsat system, which also include Emergency Locator Transmitters (ELT);

4. Satellite Tracking System (STS) Subnetworks - They can integrate STS via Inmarsat or Iridium networks, such as project of Global Aircraft Tracking (GAT) system, and here can be included maritime units known as Search and Rescue Transponder (SART) electronic device that automatically reacts to the emission of a radar onboard SAR ships or aircraft;

5. Communication, Navigation and Surveillance (CNS) Subnetworks - They can be integrated in the Global Satellite Augmentation System (GSAS) known as SBAS using GNSS-1 networks of the US GPS and the Russian GLONASS networks. These subnetworks have to include GNSS Augmentation VDL (GAVDL) and GNSS Augmentation SDL (GASDL) for more accurate and effective aeronautical CNS solutions in the flight corridors, approachings and on the airport surface; and 


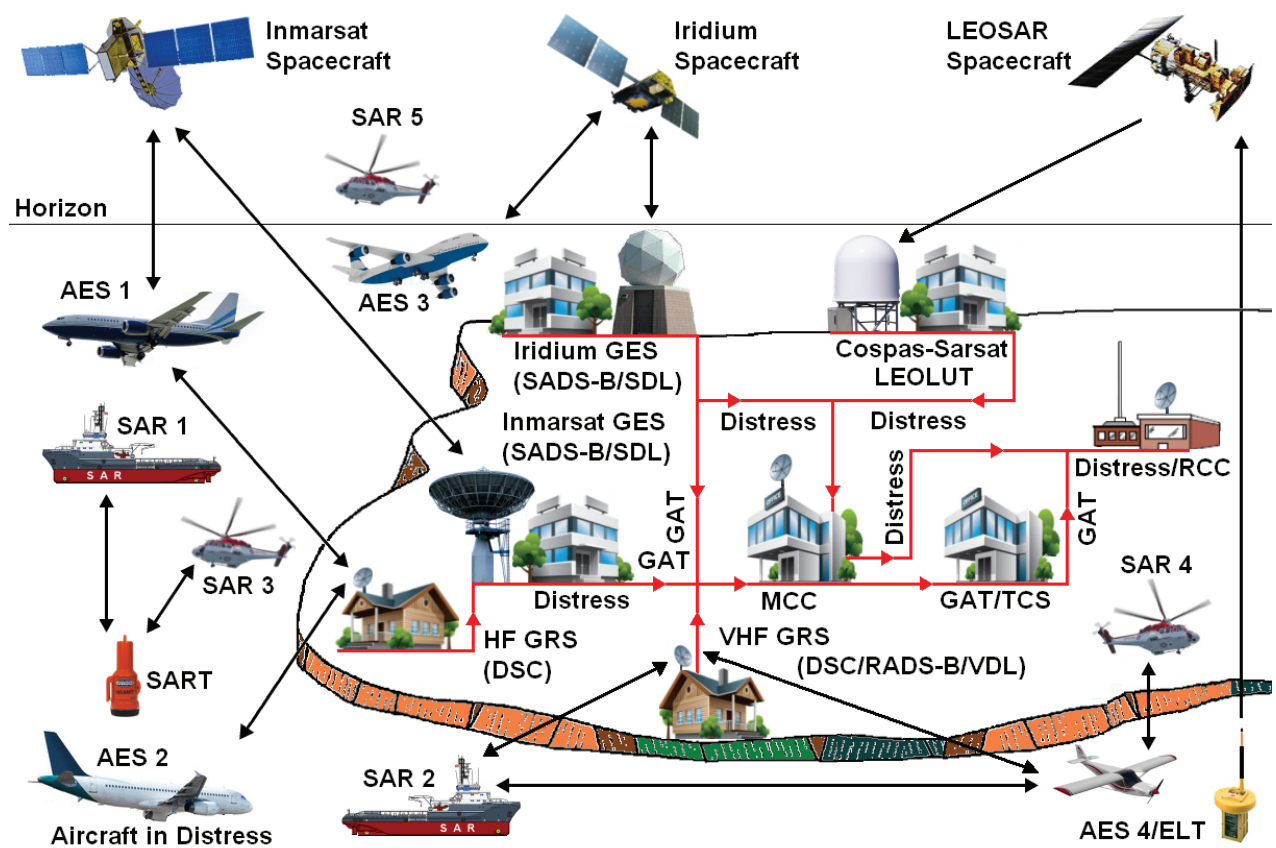

Figure 1. Concept of GADSS Space and Ground Segments - Courtesy of Book: by Ilcev (2017)

6. Stratospheric Platforms Systems (SPS) - In the stage SPS stations can be implemented for local and regional aeronautical CNS (Ilcev, 2017; Calcutt \& Tetley, 1994; Forssell, 2008).

\section{GADSS Network Configuration}

The GADSS space and ground network is developed and designed by author of this book in 2000, namely 16 years before ICAO, which integrated architecture is illustrated in Figure 1. The main components of GADSS network are VHF and HF Radio, Satellite Communications and Satellite SAR Networks. Radio system will provide service via VHF and HF bands such as DSC, VDL and RADS$B$ transmissions. In addition, Inmarsat and Iridium networks provide SADS-B and SDL via Aircraft Earth Station (AES), which will be introduced in the next sections.

The new GADSS network and technology solutions will be simplifies and affect in the near future every individual onboard aircraft. This scenario also has to be very important for aircraft crew, because in case that pilot is unable to provide distress alert via adequate onboard or auxiliary portable equipment, it can be conducted by any crewmember for saving of crew and passenger lives. However, the major importance of the GDASS network and equipment will be to provide enhanced Safety of Lives in Distress (SOLID) of aircraft incidents at sea and on the ground.

The GADSS network has to be an international infrastructure that will use integrated radio and satellite equipment and systems to ensure rapid and automated distress alerting of ground-based communication and SAR authorities for the event of an aircraft in emergency on the ground. However, any aircraft landed at sea has to be treated like ship in distress, so except alerting of ground- based SAR authorities, this network has also to alert all ships in the immediate vicinity of aircraft in distress at sea. The basic concept is that SAR authorities ashore, as well as shipping in the immediate vicinity of the aircraft in distress at sea, will be rapidly alerted through radio and satellite communication so that they can assist in a cocoordinated SAR operation with the minimum of delay.

The main aeronautical systems and equipment implemented in GADSS will be integrated Aeronautical Radio Distress and Safety Systems (ARDSS) and Aeronautical Satellite Distress and Safety Systems (ASDSS). Aircraft fitted with GADSS equipment will be safer in flight and in case of landing at sea or on the ground, and more likely to receive assistance in the event of a distress alerts (Ilcev, 2013; Stacey, 2008; Del Re \& Ruggieri, 2008; Myron \& Fried, 1997).

The GADSS network has to provide manual alerting and also automatic alerting for situation when aircraft's staff does not have time to send out a full distress call. The GADSS also requires aircraft to receive broadcasts of Aeronautical Safety Information (ASI) for any fly navigation

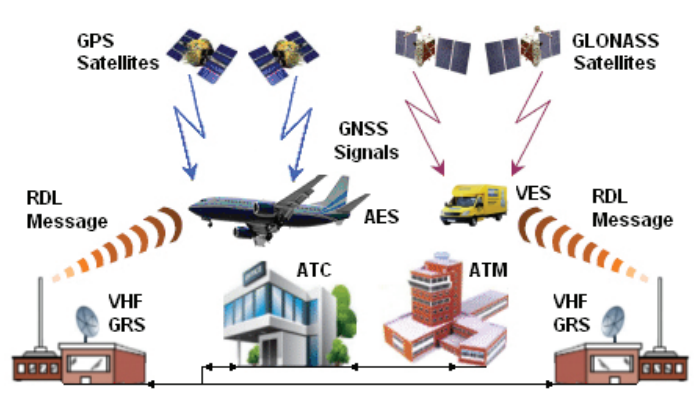

Figure 2. Aeronautical VDL Network - Courtesy of Manual: by Ilcev (2011) 
or weather warnings, and requires each aircraft to carry satellite ELT, including even maritime Emergency Position Indicator Radio Beacon (EPIRB) and Search and Rescue Transponder (SART) devices, which float free at sea and can alert SAR authorities similar to the a ship in distress.

The GADSS regulations have to be adopted by the ICAO Council, while for requirements of amendments to the International Implementation of the future GADSS will be the responsibility each country as a Contracting Governments to SOLID capacity and capability of on scene communications. In such a way, the Administrations of individual member countries have to ratify the GADSS requirements into their national law. In practice, this also means that individual aircraft owners have to be responsible for ensuring their aircraft meet GADSS requirements, since they must obtain certificates from their flag State certifying conformity with all relevant ICAO regulations and recommendations.

Therefore, according to new GADSS infrastructure, SOLID and Future Aeronautical Communications (FAC) concept, each aircraft or helicopter has to carry onboard by compulsory adequate Radio VHF/UHF and HF communication devices and Satellite equipment for Commercial and Safety operations in the case of distress alert or emergency. Namely, to provide airman concept of new projected GADSS on the road, ICAO team has to engage worldwide experts in CNS with complete experience in Radio and Satellite CNS networking.

In a more general sense, new proposed concept of GADSS satellite communication system will include Inmarsat, Iridium and three Cospas-Sarsat subsystems, such as LEOSAR, GEOSAR and MEOSAR. In this configuration will be included new proposals for HEO Molniya, O3b and new Global Aircraft Tracking (GAT) as SAR satellite subsystems. Here will be shortly introduced only four GADSS systems such as: VDL, RADS-B, SDL and SADS-B, while about GAT and other GADSS systems reader can find in references (Ilcev, 2011, 2013, 2014).

\subsection{Aeronautical VHF Data Link (VDL) Network}

The aeronautical VHF Data Link 4 (VDL4) radio communication mode was originally conceived and planned as a broadcast link used to support navigation and surveillance functions. In fact, aeronautical navigation data link is used to send GNSS corrections to the GNSS Receiver (Rx) in order to increase the position accuracy of aircraft along with GNSS signals integrity.

Surveillance data link is using new concepts (Mode $S$ or ADS-C) or emerging concepts (RADS-B, TIS-B or FISB) based on the broadcast of aircraft flight information through data links in order to increase the traffic efficiency and safety in the air space.

The special aeronautical VDL transponders for determination and traffic management can be installed onboard aircraft flying in approaching areas to airports and movements through the airport surface, which scenario is shown in Figure 2.

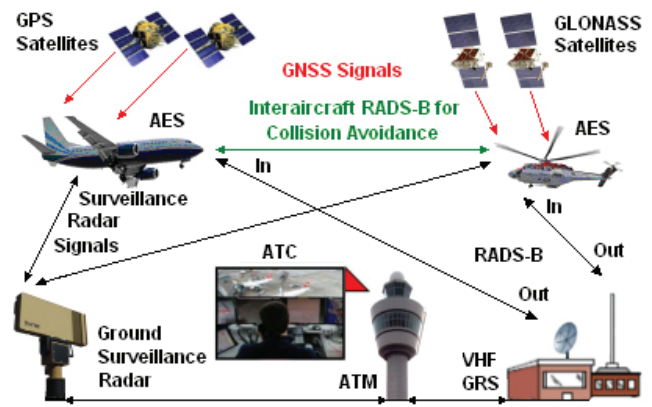

Figure 3. Aeronautical RADS-B Network - Courtesy of Manual: by Ilcev (2011)

In the same way, land vehicle can carry onboard the similar VDL transponders for traffic management of vehicle movements inside airports. The system is working in the way that VDL equipment onboard aircraft or vehicle is getting GNSS signals from GPS or GLONASS satellites and sending own position via VHF Ground Radio Station (GRS) to the ATC and ATM.

\subsection{Aeronautical Radio Automatic Dependent Surveillance-Broadcast (RADS-B)}

This VHF mode provides in and out RADS-B radio transmissions from aircraft, approximately once a second, Position, Velocity and Time (PVT), altitude and flight identity, illustrated in Figure 3. Besides, aircraft can receive signals from Ground Surveillance Radar and RADS-B can provide links for exchange PVT data for collision avoidance between aircraft flying in VHF coverage.

Typically, the airborne onboard position sensor is a GNSS receiver (GPS or GLONASS), or the GNSS output of an MMR terminal. An RADS-B GRS uses a non-rotating omni-directional antenna to receive messages transmitted by the aircraft. The RADS-B equipment is designed as a multiple use surveillance technique for aerodrome surface facilities, terminals and en-route airspace and is applicable to both ATC and aircraft-to-aircraft surveillance. Thus, RADS-B is "line of sight" based surveillance that requires GRS to receive data and transmit to Aircraft Traffic Management (ATM). A single GRS can provide coverage out to approximately $250 \mathrm{Nm}$ for enroute, terminals and aerodrome surface surveillance system. At this point, RADS-B requires new equipage for aircraft and RADS-B accuracy and integrity is subject to the source of the navigation data (usually GNSS). The RADS-B application offers benefits in:

a) Extending existing coverage to any hypothetical areas of Flight Information Regions (FIR) not currently covered by radar surveillance including aerodrome surface surveillance;

b) Providing redundancy to existing surveillance systems;

c) Increasing surveillance accuracy and consistency in tracking;

d) Facilitating a reduction in capital and maintenance costs for surveillance infrastructure; 
e) Providing a starting point for airborne spacing and separation procedures; and

f) Increase safety, capacity and efficiency within FIR area for all equipped airspace users.

The ICAO team has identified RADS-B as a main component in future ATM surveillance and is actively supporting RADS-B implementation.

\subsection{Aeronautical Satellite Data Link (SDL) Network}

The concept of aeronautical SDL service is similar to VDL Mode 4 system, which is able to provide a satellite broadcast link supporting navigation and surveillance functions for aircraft and airport vehicles. The SDL can provide transmission of GNSS signals received from GPS or GLONASS satellites by AES and Vehicle Earth Stations (VES), and sent via GEO or LEO satellites as SDL messages to the Ground Earth Stations (GES) ATC and ATM.

In the other words, the SDL network is a part of total aeronautical satellite communication configuration that provides very important Aeronautical SDL via GEO Inmarsat or LEO Iridium constellation. The data link operates at $200 \mathrm{~b} / \mathrm{s}$, uses Forward Error Correction (FEC) coding and employs a terminal monitor that provides interfaces to onboard avionics data recording equipment or even onboard airport vehicles and an industry-standard Personal Computer (PC) system. The PC terminal serves as a user terminal as well as a real-time monitor of BitError-Rate (BER) performance.

The SDL network is a part of total aeronautical communication solutions for:

1. SDL Tracking Messages Service - The SDL system can provide transmission of Short Burst Messages (SBM) between mobile stations or mobile units with GES, ATC and ATM, which scenario is shown in Figure 4. In mobiles, such as aircraft and surface vehicles, can be installed satellite transponders or satellite tracker devices, which are getting GNSS signals from GPS or GLONASS spacecraft. Mobile transponders can send PVT and other data via any GES covering Inmarsat or Iridium satellites to ATC and ATM centres. Thus, the SDL transponder can support the similar services that provide VDL4, but using Iridium network it can provide real global coverage including both poles, while Inmarsat can provide near global coverage

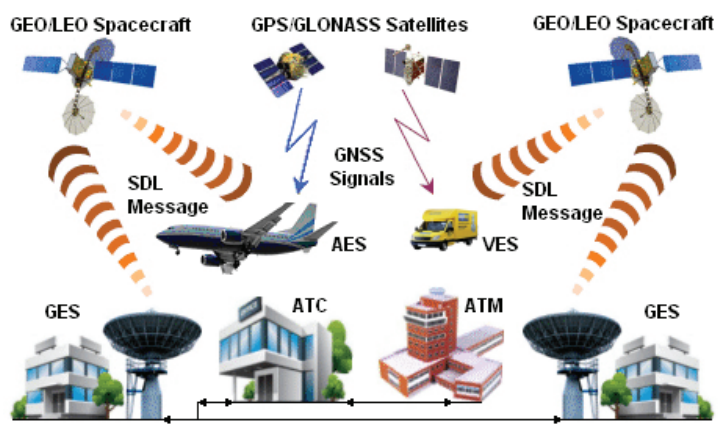

Figure 4. Aeronautical SDL Network - Courtesy of Book: by Ilcev (2013) without poles. The transponder allows pilots and air traffic controllers to "display" aircraft traffic in the air and on the airport surface including vehicle movements with the highest possible precision. The GES units can easily interface with other surveillance systems through the standardized Asterix protocol, enabling a complete surveillance picture at the airport derived from several sources. Ground network will provide increased functionality and capability for wide area coverage of advanced ATM applications. The functionality of the ground station is tailored to system specific services by its software configuration.

2. SDL of SBM and High Speed Data (HSD) Service Every aircraft and helicopter caring transponders or satellite communication devices will be able to send and receive $\mathrm{SBD}$ or $\mathrm{HSD}$ two-ways text messaging for CNS purposes, flight movement data, text and graphical weather, NOTAM alerts and in-flight route planning, which are made possible by Inmarsat and Iridium satellite services (Ilcev, 2016; Stacey, 2008; Del Re \& Ruggieri, 2005).

\subsection{Aeronautical Satellite Automatic Dependent Surveillance}

The SADS-B network is a new system in deployment phase for airborne mission similar to RADS-B network, with the only difference that it operates via GEO or LEO satellite constellations instead of the conventional VHF radio. Thus, this system is a modern satellite broadcasting from aircraft via satellites and GEO terminals to provide position, velocity, altitude, positional integrity, flight identity, 24-bit aircraft address and other data that have been detected and computed by onboard aircraft sensors.

This SADS-B system will provide PVT and other data detected and computed by onboard ships sensors, such as GNSS (GPS or GLONASS), radar and other instruments. On 23 May 2013 a German DLR trial was switched on for the first time onboard A320, recording over 12.000 ADS-B messages within two hours at an altitude of 820 kilometers.

Typical SADS-B aeronautical network is similar to the airborne RADS-B with additional differences that the SADS-B network is covering long distances and is using transmission service of GEO or LEO satellites to

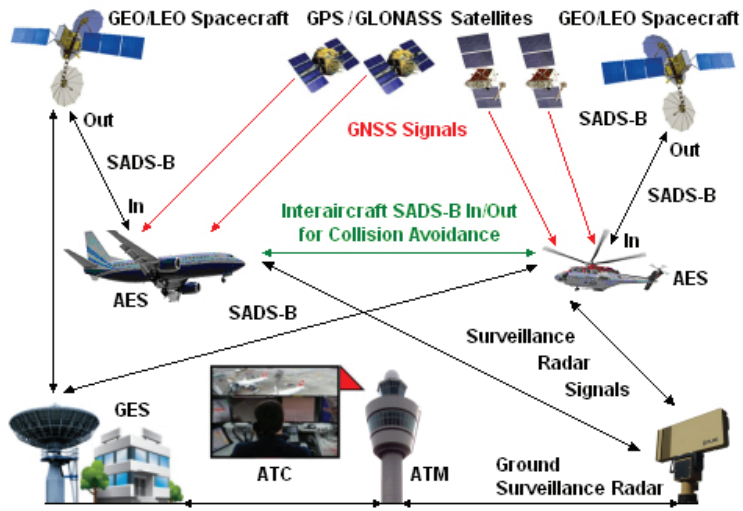

Figure 5. Aeronautical SADS-B System - Courtesy of Book: by Ilcev (2013) 
send OUT or receive IN SADS-B information to STC and STM via GES terminals, which configuration is shown in Figure 5. The SADS-B can provide the following service within avionic routes in ocean areas and approachings for enroute opeartions:

1. Air-to-air transmission implies data broadcast from one aircraft with the possibility of the reception and display of data in other aircraft and vice versa;

2. Air-to-ground transmission implies data broadcast from an aircraft with the possibility of reception and display in certain ATC units; and

3. Ground-to-air transmission implies data broadcast from the ground with the possibility of reception and display onboard certain aircraft.

Therefore, an SADS-B is a surveillance in which an aircraft determines its position via satellite navigation and periodically broadcasting signals. This data can be received and send by interaircarft communication IN/ OUT for enhanced collision avoidance and can be also received by ATC as a replacement for airborne ground radar system. Otherwise the ground surveillance radars can be used as back up to SADS-B system. The SADS-B system requires new equipage for aircraft and SADS-B accuracy and integrity is subject to the source of the navigation data (usually GNSS).

In addition, AES can receive signals from Ground Surveillance Radar and SADS-B can provide IN and OUT links for exchange PVT data for collision avoidance between aircraft flying in GEO or LEO satellite coverage (Ilcev, 2017; Myron \& Fried, 1997; Prasad \& Ruggieri, 2005).

\section{Aeronautical Global Aircraft Tracking (GAT) Network}

The Long Range Identification and Tracking (LRIT) is compulsory system onboard ships established by IMO on 19 May 2006, as solution for global ships tracking. However, before that, author of this book in 2000 proposed to IMO his Global Ship Tracking (GST) as better solution and with more convenient designation than LRIT. The previous SADS-B system has not some features as GAT does such as:

1. This system is not discrete so that someone uninvited, under force by pirates or purposly can turn off the unit completely, part of the unit or just GNSS receiver;

2. This system cannot work properly if it has not an integrated GNSS receiver; and

3. This system needs to be installed to some secret place and although is powered by aircraft sources it needs own charger and batteries.

As stated at the beginning of this chapter, earlier in 2000 author of this book proposed to ICAO unique project known as Global Aircraft Tracking (GAT). This project is the best and only solution for aircraft tracking in real time and space, which diagram is depicted in Figure 6. In the same way as maritime GST network, an aircraft in flight is receiving GNSS signals from GPS or GLONASS spacecraft by its GAT equipment and then is sending PVT data

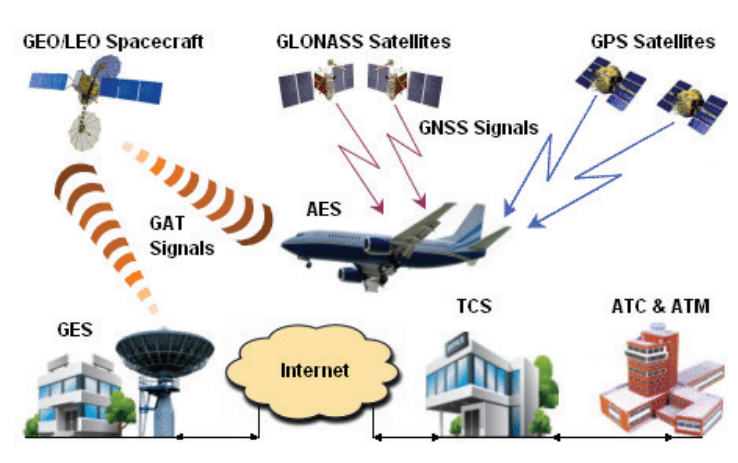

Figure 6. Aeronautical GAT System for GASDL Network Courtesy of Book: by Ilcev (2017)

to Tracking Control Station (TCS) via GES and Internet. The TCS unit is connected to ATC and ATM terminals for eventual coordination in SAR, tracking and collision avoidance. Thus, the PVT data of GAT signals as messages include the airborne equipment identifier, altitude, positioning PVT data of latitude and longitude including the date and time of the transmission. The system has to specify that flag States should ensure a minimum of 30 to 60 GAT messages daily per hour are sent, though the frequency of messages can be changed to a minimum of once every 5 minutes through an user request.

The TCS is de facto brain of the GAT system, which is receiving PVT and other data sent by GAT units from GES via Inmarsat or Iridium satellites, and then its Processing Data Centre (PDC) is processing and memorizing this data. The TCS units have to be distributed in each FIR or smaller flight arrears and to be connected to nearest ATC/ATM. The TCS terminal will display all processed GAT data on like radar display showing position of all aircraft in certain flight area. In case of any incident, TCS terminal will send all necessary particulars about certain aircraft in distress or emergency to the SAR forces, which will find it in few days and in radius of several tenths of nautical miles. Thus, in the future is not acceptable to have situation similar as Air France and Malaysian aircraft anymore.

Furthermore, the GAT unit onboard aircraft will be able to receive PVT data of all aircraft flying in certain area and be used for enhanced collision avoidance. The GAT receiver unit can be connected to the special display with keyboard in cockpit showing PTV data of all adjacent airplanes during flight. As stated earlier, all operations of GAT transceiver are automatic, so cannot be controlled by pilot or any operator at all, but it can be connected to laptop or palmtop, so pilot will be able to send own PTV report to TCS and to receive or poll data in return. This solution is also very important tool during extremely bad weather conditions, thunderstorms and very poor visibility.

The GAT system has to develop three segments: Space, Ground and Users and to provide complete GAT Network. In fact, GAT message can be sent via Inmarsat and Iridium (if aircraft is flying over the North Pole) communications satellite. Ground infrastructures are GES terminals, 
Internet or TTN and TCS with PDC, while users are all type of commercial aircraft and helicopters.

The PDC facilities should store all incoming GAT information received from aircraft and distributed this data to different users according to the GAT Data Distribution Plan (DDP). Otherwise, the PVT data users can be airways companies, ATC and ATM units, regional SAR forces and any aircraft flying in area of certain TCS for purpose of enhanced collision avoidance. Therefore, the GAT unit is presenting digital transmission system that provides automatically messaging or reporting of PTV data and polling data from TCS as well.

The innovative GAT is better than ACARS and ADS-B because is discrete, independent and has own power supply. In fact, the GAT unit has to be installed onboard aircraft secretly, i.e. discrete, and in such a way to protect accidental or forced shutdown of the device as a whole. The GAT unit is always ON and programmed to receive GPS data and to transmit SBD message via satellite and GES to the PDC unit of TCS. In any emergency or distress situation, TCS will send PVT data of aircraft to SAR forces, which than will participate in SAR operations.

The GAT unit can be installed in any small or different longhoul jets and rotarywing aircraft without any additional software to upgrade of an existing system and does not need modification of ground and/or satellite equipment. In fact, GAT unit with antenna is totally new aircraft hardware with firmware and has to be installed discrete below fuselage together with batteries and interfaced to onboard powers supply. The Space Science Centre (SSC) leaded by author of this book has complete GAT installation for a trial, looking for interested companies and individuals for collaboration (Ilcev, 2013; Prasad \& Ruggieri, 2005; Kaplan, 1996).

\section{Conclusions}

Without consideration of the main subject of ICAO FANS, the best solution will be the establishment of Future GADDS separately, more effective and similar to a current GMDSS of IMO. Thus, the GADSS network needs integration of satellite and radio networks, in which can be included Inmarsat, Iridium and other mobile satellite networks; Cospas-Sarsat LEOSAR, MEOSAR and GEOSAR networks with ELT, Personal Locator Beacons (PLB) or EPIRB radio beacons; and $\mathrm{VHF} / \mathrm{HF}$ radio networks with
Digital Selective Call (DSC) system. Without doubt, the new developed Global Aircraft Tracking (GAT) by Space Science Centre (SSC) at DUT, VDL, SDL, RADS-B, SADS$\mathrm{B}$ and Cospas-Sarsat radio beacons have to be mandatory onboard every aircraft. Even maritime EPIRB can be installed onboard aircraft, in case of any emergency landing at sea, owing to engine or other troubles, or to use as well as ELT with floating possibilities. Following this situation, the SAR procedure for aircraft floating on the sea's surface has to be same as for ships in distress.

In any event, the development of new missions for GADSS has to be led by the ICAO and supported by other communities and researchers involved in AMSC services, including Inmarsat, Iridium and Cospas-Sarsat satellite systems. Without the new GADSS integrated mission of radio and satellite CNS systems, all other solutions, technology transfers, innovations and technical implementations will not be complete and successful.

\section{References}

Calcutt, D., \& Tetley, L. (1994). Understanding GMDSS. London: Edward Arnold.

Del Re, E., \& Ruggieri, M. (2008). Satellite communications and navigation systems. New York: Springer.

https://doi.org/10.1007/978-0-387-47524-0

Forssell, B. (2008). Radionavigation systems. Boston-London: Artech House.

Global Aeronautical Distress and Safety System (GADSS) (2002). CNS system, Durban.

Ilcev, D. S. (2017). Global mobile satellite communications for maritime, land and aeronautical applications, theory and applications (Vols 1 and 2). Boston: Springer.

Ilcev, D. S. (2013). Global aeronautical Communications, Navigation and Surveillance (CNS), theory and applications (Vols 1 and 2, AIAA). Reston.

Ilcev, D. S. (2011). Global Aeronautical Distress and Safety System (GADSS). Durban: Durban University of Technology.

Ilcev, D. S. (2014). Global Radio and Satellite CNS. Durban: Durban University of Technology.

Kaplan, E. D. (1996). Understanding GPS principles and applications. Boston: Artech House.

Myron, K., \& Fried, W. R. (1997). Avionics navigation systems. John Wiley \& Sons, Ltd.

Prasad, R., \& Ruggieri, M. (2005). Applied satellite navigation using GPS, GALILEO, and augmentation systems. Boston: Artech House.

Stacey, D. (2008). Aeronautical radio communication systems and networks. Chichester: John Wiley \& Sons, Ltd. https://doi.org/10.1002/9780470035108 